\title{
AIZENMAN'S THEOREM FOR ORTHOGONAL POLYNOMIALS ON THE UNIT CIRCLE
}

\author{
BARRY SIMON*
}

\begin{abstract}
For suitable classes of random Verblunsky coefficients, including independent, identically distributed, rotationally invariant ones, we prove that if

$$
\mathbb{E}\left(\int \frac{d \theta}{2 \pi}\left|\left(\frac{\mathcal{C}+e^{i \theta}}{\mathcal{C}-e^{i \theta}}\right)_{k \ell}\right|^{p}\right) \leq C_{1} e^{-\kappa_{1}|k-\ell|}
$$

for some $\kappa_{1}>0$ and $p<1$, then for suitable $C_{2}$ and $\kappa_{2}>0$,$$
\mathbb{E}\left(\sup _{n}\left|\left(\mathcal{C}^{n}\right)_{k \ell}\right|\right) \leq C_{2} e^{-\kappa_{2}|k-\ell|}
$$

Here $\mathcal{C}$ is the CMV matrix.
\end{abstract}

\section{INTRODUCTION}

This paper is a contribution to the theory of orthogonal polynomials on the unit circle (OPUC); for background on OPUC, see Szegő [19], Geronimus [7, and Simon [15, 16]. Our goal here is to prove an analog of a result of Aizenman [1] for random discrete Schrödinger operators. Aizenman considers operators on $\ell^{2}\left(\mathbb{Z}^{\nu}\right)$ of the form $h_{\omega}=h_{0}+V_{\omega}$ where (1] allows more general $h_{0}$ than this!)

$$
\left(h_{0} u\right)(n)=\sum_{|j|=1} u(n+j)
$$

and $V_{\omega}$ is the diagonal matrix whose matrix elements are independent identically distributed random variables. Aizenman's theorem states

Theorem (Aizenman [1]). Under suitable hypotheses on the distribution of $V$, if for some $[a, b] \subset \mathbb{R}$,

$$
\int_{a}^{b} \mathbb{E}\left(\left|\left[\left(h_{\omega}-E-i 0\right)^{-1} P_{[a, b]}\left(h_{\omega}\right)\right]_{k \ell}\right|^{p}\right) d E \leq C_{1} e^{-\kappa_{1}|k-\ell|}
$$

for some $0<p<1$ and $\kappa_{1}>0$, then for some $\kappa_{2}>0$ and $C_{2}$,

$$
\mathbb{E}\left(\sup _{t}\left|\left[e^{-i t h_{\omega}} P_{[a, b]}\left(h_{\omega}\right)\right]_{k \ell}\right|\right) \leq C_{2} e^{-\kappa_{2}|k-\ell|}
$$

Date: September 27, 2004.

2000 Mathematics Subject Classification. 26C05, 82B44, 47N20.

Key words and phrases. OPUC, random Verblunsky coefficients, localization.

* Mathematics 253-37, California Institute of Technology, Pasadena, CA 91125. E-mail: bsimon@caltech.edu. Supported in part by NSF grant DMS-0140592. 
Aizenman's motivation was that Aizenman-Molchanov [2] had proven bounds of the form (1.1) (generally called Aizenman-Molchanov or fractional moment bounds) realizing that the key was to restrict $p$ to be less than 1. From their bounds, they easily obtained spectral localization (i.e., pure point spectrum) by using the Simon-Wolff criterion [17. Aizenman was interested in (1.2) because it is a form of physical localization. It was used by del Rio et al. 5] to obtain what is now the standard strong form of eigenfunction localization (SUDL) and by Minami 12 to prove Poisson distribution of the eigenvalues of $h_{\omega}$ restricted to a large box. Del Rio et al. also simplified Aizenman's proof and slightly extended the result (so that the theorem we stated above is their form with some extra hypotheses dropped).

To describe precisely the result we want to prove here, we need some preliminaries. Given a set of Verblunsky coefficients, $\left\{\alpha_{j}\right\}_{j=0}^{\infty}$ (see [15, Section 1.5]), one forms the CMV matrix, $\mathcal{C}$ (see Cantero-Moral-Velázquez [4] or [15. Chapter 4]). We define, for $z \in \mathbb{D}$, the unit disk

$$
F_{k \ell}(z)=\left[\frac{\mathcal{C}+z}{\mathcal{C}-z}\right]_{k \ell}
$$

By Kolmogorov's theorem (see [1] or Duren [6, Section 4.2]), $F_{k \ell}$ lies in the Hardy spaces $H^{p}(\mathbb{D})$ for $0<p<1$, so

$$
F_{k \ell}\left(e^{i \theta}\right) \equiv \lim _{r \uparrow 1} F_{k \ell}\left(r e^{i \theta}\right)
$$

exists for $\frac{d \theta}{2 \pi}$-a.e. $\theta$ and has an integrable $p$-th power over $\partial \mathbb{D}$ for $p \in(0,1)$.

Now let the $\alpha$ 's be random variables which define a measure $d \Gamma$ on $\times_{j=0}^{\infty} \mathbb{D} \equiv \mathbb{D}^{\infty}$. For each $n=0,1,2, \ldots$ and $\lambda \in \partial \mathbb{D}$, define $T_{n, \lambda}: \mathbb{D}^{\infty} \rightarrow \mathbb{D}^{\infty}$ by

$$
\begin{aligned}
\left(T_{n, \lambda}(\alpha)\right)_{j} & =\alpha_{j} & & j=0,1, \ldots, n-1 \\
& =\lambda \alpha_{j} & & j=n, n+1, \ldots
\end{aligned}
$$

and let $d \Gamma_{n, \lambda}(\alpha)=d \Gamma\left(T_{n, \lambda}(\alpha)\right)$. We say $d \Gamma$ is strongly quasi-invariant if each $d \Gamma_{n, \lambda}$ is $d \Gamma$-absolutely continuous and $\sup _{n, \lambda}\left\|d \Gamma_{n, \lambda} / d \Gamma\right\|_{\infty}<\infty$. Clearly, if $d \Gamma$ is a product of rotation invariant measures (invariant i.i.d.'s), $d \Gamma$ is strongly quasi-invariant. We will discuss other examples in Section 7 . Here is our main result:

Theorem 1.1. Suppose $\left\{\alpha_{j}\right\}_{j=0}^{\infty}$ are random Verblunsky coefficients which are strongly quasi-invariant, and for some $p<1$ and $\kappa_{1}>0$,

$$
\mathbb{E}\left(\int_{0}^{2 \pi}\left|F_{k \ell}\left(e^{i \theta}\right)\right|^{p} \frac{d \theta}{2 \pi}\right) \leq C_{1} e^{-\kappa_{1}|k-\ell|}
$$

Then for suitable $\kappa_{2}>0$ and $C_{2}$,

$$
\mathbb{E}\left(\sup _{n}\left|\left(\mathcal{C}^{n}\right)_{k \ell}\right|\right) \leq C_{2} e^{-\kappa_{2}|k-\ell|}
$$


Remarks. 1. This result is interesting only because one can prove (1.6). For certain cases of rotation invariant i.i.d. $\alpha$ 's, Stoiciu [18] has proven (1.6). Indeed, I proved Theorem 1.1precisely to fill in a missing step in his program to prove Poisson distribution for the zeros of paraorthogonal polynomials with random Verblunsky coefficients.

2. Kolmogorov's argument proves for any OPUC, any $k, \ell$, and $0<p<1$,

$$
\int_{0}^{2 \pi}\left|F_{k \ell}\left(e^{i \theta}\right)\right|^{p} \frac{d \theta}{2 \pi} \leq 2 \cos \left(\frac{p \pi}{2}\right)
$$

This means that if (1.6) holds for one $p \in(0,1)$, it holds for all such $p$ (with $\kappa_{1}$ dependent on $p$ ).

3. The $\sup _{n}$ is over $n=0, \pm 1, \pm 2, \ldots$.

4. (1.7) is a strong statement about the structure of eigenfunctions of $\mathcal{C}$ and of OPUC to be compared with the case $\alpha \equiv 0$ where $\sup _{n}\left|\left(\mathcal{C}^{n}\right)_{k \ell}\right|=1$.

5. If (1.6) holds for $p=1$ (it cannot, as we will see in Remark 6!), (1.7) would be immediate since $2\left(\mathcal{C}^{n}\right)_{k \ell}$ are the Taylor coefficients of $F_{k \ell}$. Thus, (1.6) $\Rightarrow$ (1.7) without recourse to the expectations. But, in general, for $p<1, H^{p}$ functions have Taylor coefficients that can grow as $o\left(n^{1 / p-1}\right)$ and no better (see Duren [6, Chapter 6]), so (1.6) $\Rightarrow$ (1.7) only holds because the $\sup \left|\left(\mathcal{C}^{n}\right)_{k \ell}\right|$ is averaged over a set of rank one perturbations. This is Aizenman's key discovery in [1].

6. (1.6) cannot hold for $p=1$. Indeed, if $\sum_{\ell}\left(\int_{0}^{2 \pi}\left|F_{k \ell}\left(e^{i \theta}\right)\right| \frac{d \theta}{2 \pi}\right)^{2}<\infty$ for fixed $k$, then $\sum_{\ell}\left|\mathcal{C}_{k \ell}^{n}\right|^{2} \rightarrow 0$ by appealing to the dominated convergence theorem for sums and the Riemann-Lebesgue lemma which implies $\left(\mathcal{C}^{n}\right)_{k \ell} \rightarrow$ 0 if $\int\left|F_{k \ell}\left(e^{i \theta}\right)\right| \frac{d \theta}{2 \pi}<\infty$. But since $\mathcal{C}^{n}$ is unitary, $\sum_{\ell}\left|\mathcal{C}_{k \ell}^{n}\right|^{2}=1$.

While part of our proof of Theorem 1.1 follows the arguments in del Rio et al. [5], there are two novel aspects that prompted me to write this separate note. The first involves the theory of rank one perturbations. This theory is well-developed for selfadjoint operators (see 3, 14]), but I could not find any extensive theory for the unitary case when I wrote [15, 16, so I developed the theory there (see [15, Subsections 1.3.9, 1.4.16, and Section 4.5]). It turns out that a key formula needed here (see (2.14) below) is not in that presentation.

Secondly, OPUC has a subtlety missing from the Schrödinger case. Namely, the relevant rank one perturbations of the Schrödinger operators are also Schrödinger operators, but the rank one perturbations of CMV matrices are not CMV matrices. Of course, as unitary matrices with a cyclic vector, these are unitarily equivalent to CMV matrices and, as we will see, a formula of Khrushchev [10] even implies what the Verblunsky coefficients are for the new matrices. But we will need to know the form of the unitary, and this will require an illuminating calculation that should be useful in other contexts. 
In Section 2 we discuss some aspects of the theory of rank one perturbations of unitaries, which we use in Section 3, following [1, 5, to compute explicit spectral representations. In Section 4, we use this to obtain a deterministic form of Aizenman's theorem that involves averaging under rank one perturbations, and in Section 5, we write these rank one perturbations in terms of CMV matrices. Section [6 puts everything together to get Theorem 1.1 and Section 7 has some comments.

I would like to thank Mihai Stoiciu for useful discussions.

\section{Rank One Perturbations of Unitaries Revisited}

Rank one perturbations of unitaries are best understood multiplicatively. Let $U$ be a unitary operator on a Hilbert space, $\mathcal{H}$, and $\varphi \in \mathcal{H}$ a unit vector. Let $P=\langle\varphi, \cdot\rangle \varphi$ be the projection onto the multiples of $\varphi$. One defines for $\lambda \in \partial \mathbb{D}$,

$$
U_{\lambda}=U(1-P)+\lambda U P=U[(1-P)+\lambda P]
$$

(if $\varphi$ is part of an orthonormal basis, $(1-P)+\lambda P$ is the diagonal matrix with $\lambda$ in $\varphi$ position and 1 in all others). Thus

$$
\begin{aligned}
& U_{\lambda} \varphi=\lambda U \varphi \\
& U_{\lambda} \psi=U \psi \quad \text { if } \psi \perp \varphi
\end{aligned}
$$

which also defines $U_{\lambda}$. Note also that

$$
U_{\lambda}-U=(\lambda-1) U P
$$

For $z \in \mathbb{D}$ and $\|\varphi\|=1$, define

$$
F_{\varphi}(z)=\left\langle\varphi, \frac{U+z}{U-z} \varphi\right\rangle
$$

which is a Carathéodory function (i.e., $F(0)=1$ and $\operatorname{Re} F>0$ on $\mathbb{D}$ ). Since

$$
\left\langle\varphi, \frac{U-z}{U-z} \varphi\right\rangle=1
$$

we can solve for

$$
\begin{aligned}
\left\langle\varphi, U(U-z)^{-1} \varphi\right\rangle & =\frac{1}{2}\left[F_{\varphi}(z)+1\right] \\
\left\langle\varphi,(U-z)^{-1} \varphi\right\rangle & =\frac{1}{2 z}\left[F_{\varphi}(z)-1\right]
\end{aligned}
$$

Notice (2.7) and (2.8) hold if $U$ is replaced by $U_{\lambda}$ and $F_{\varphi}$ by $F_{\varphi}^{\lambda}(z)$ given by (2.5) with $U$ replaced by $U_{\lambda}$. By the second resolvent equation,

$$
\begin{aligned}
\left(z-U_{\lambda}\right)^{-1} U \varphi & =(z-U)^{-1} U \varphi+(z-U)^{-1}\left(U_{\lambda}-U\right)\left(z-U_{\lambda}\right)^{-1} U \varphi \\
& =\left[1+(\lambda-1)\left(\varphi,\left(z-U_{\lambda}\right)^{-1} U \varphi\right)\right](z-U)^{-1} U \varphi
\end{aligned}
$$

on account of (2.4). 
Taking an inner product of (2.9) with $\varphi$ and using (2.7)/(2.8) for $U$ and $U_{\lambda}$ let us solve for $F_{\varphi}^{\lambda}(z)$ in terms of $F_{\varphi}(z)$. The result (see 15, Subsection 1.4.16]) is expressed most succinctly via the function $f$ defined by

$$
F_{\varphi}(z)=\frac{1+z f(z)}{1-z f(z)}
$$

for then the inner product of $\varphi$ with (2.9) implies

$$
F_{\varphi}^{\lambda}(z)=\frac{1+\lambda^{-1} z f(z)}{1-\lambda^{-1} z f(z)}
$$

Notice, by (2.7)/(2.8), that

$$
\begin{aligned}
\left\langle\varphi, U_{\lambda}\left(U_{\lambda}-z\right)^{-1} \varphi\right\rangle & =\frac{1}{1-\lambda^{-1} z f(z)} \\
\left\langle\varphi,\left(U_{\lambda}-z\right)^{-1} \varphi\right\rangle & =\frac{\lambda^{-1} f(z)}{1-\lambda^{-1} z f(z)}
\end{aligned}
$$

Thus far, the formulae are identical to what is in [15. What is new here is to note that (2.9) says that as a vector in $\mathcal{H}$, for each $z \in \mathbb{D}, \lambda \in \partial \mathbb{D}$, $\left(U_{\lambda}-z\right)^{-1} U_{\lambda} \varphi$ is a multiple of $(U-z)^{-1} U \varphi$, so for any $\psi \in \mathcal{H}$,

$$
\frac{\left\langle\psi,\left(U_{\lambda}-z\right)^{-1} U_{\lambda} \varphi\right\rangle}{\left\langle\varphi,\left(U_{\lambda}-z\right)^{-1} U_{\lambda} \varphi\right\rangle}=\frac{\left\langle\psi,(U-z)^{-1} U \varphi\right\rangle}{\left\langle\varphi,(U-z)^{-1} U \varphi\right\rangle}
$$

In particular, by (2.12) and $\left\langle\psi,\left(U_{\lambda}-z\right)\left(U_{\lambda}-z\right)^{-1} \varphi\right\rangle=\langle\psi, \varphi\rangle$, we see

Proposition 2.1. If $\psi \perp \varphi$, then

$$
\left\langle\psi, \frac{U_{\lambda}+z}{U_{\lambda}-z} \varphi\right\rangle=\frac{1-z f(z)}{1-\lambda^{-1} z f(z)}\left\langle\psi, \frac{U+z}{U-z} \varphi\right\rangle
$$

Remark. (2.14) is an analog of (3.2) of Aizenman [1].

\section{The Spectral Representation}

We add two extra assumptions to our analysis of rank one perturbations of unitaries. First, we suppose $\varphi$ is cyclic for $U$, that is, $\left\{U^{k} \varphi\right\}_{k=-\infty}^{\infty}$ spans $\mathcal{H}$, in which case it is easy to see that $\varphi$ is cyclic for $U_{\lambda}$. The spectral theorem then implies there are spectral measures, $d \mu_{\lambda}$, on $\partial \mathbb{D}$ defined by

$$
\int \frac{e^{i \theta}+z}{e^{i \theta}-z} d \mu_{\lambda}(\theta)=F_{\varphi}^{\lambda}(z)
$$

for $z \in \mathbb{D}$ and unique unitary maps $\mathcal{F}_{\lambda}: \mathcal{H} \rightarrow L^{2}\left(\partial \mathbb{D}, d \mu_{\lambda}\right)$, so that

$$
\left(\mathcal{F}_{\lambda} U_{\lambda} \psi\right)(z)=z\left(\mathcal{F}_{\lambda} \psi\right)(z) \quad \mathcal{F}_{\lambda} \varphi \equiv 1
$$

In particular, if $U_{\lambda} \eta_{z_{0}}=z_{0} \eta_{z_{0}}$ for some $z_{0} \in \partial \mathbb{D}$ and $0 \neq \eta \in \mathcal{H}$, then, by (3.2),

$$
\left(\mathcal{F}_{\lambda} \psi\right)\left(z_{0}\right)=\frac{\left\langle\eta_{z_{0}}, \psi\right\rangle}{\left\langle\eta_{z_{0}}, \varphi\right\rangle}
$$


Our second assumption, following [3] [17, is that for a.e. $e^{i \theta_{0}} \in \partial \mathbb{D}$,

$$
G\left(e^{i \theta_{0}}\right) \equiv \int \frac{d \mu(\theta)}{\left|e^{i \theta_{0}}-e^{i \theta}\right|^{2}}<\infty
$$

By arguments in [17, it is easy to see that if $\left\{\psi_{j}\right\}_{j=0}^{\infty}$ is a basis for $\mathcal{H}$, then (3.4) at $\theta_{0}$ is equivalent to

$$
\sum_{j=0}^{\infty} \lim _{r \uparrow 1}\left|\left\langle\psi_{j},\left(\frac{U+r e^{i \theta_{0}}}{U-r e^{i \theta_{0}}}\right) \varphi\right\rangle\right|^{2}<\infty
$$

Moreover, if (3.4) holds, then

$$
\lim _{r \uparrow 1} e^{i \theta_{0}} f\left(r e^{i \theta_{0}}\right)=\lambda_{0} \in \partial \mathbb{D}
$$

and $z_{0}$ is an eigenvalue of $U_{\lambda_{0}}$. Since (spectral averaging, due to GolinskiiNevai [8] in this setting)

$$
\int_{0}^{2 \pi}\left(d \mu_{e^{i \varphi}}(\theta)\right) \frac{d \varphi}{2 \pi}=\frac{d \theta}{2 \pi}
$$

(3.4) for a.e. $e^{i \theta_{0}}$ implies $U_{\lambda}$ has pure point spectrum for a.e. $\lambda$. (These facts are all explained in [16, Sections 10.1 and 10.2].)

Proposition 3.1. If $\varphi$ is cyclic and (3.4) holds at $\theta_{0}$, let $\lambda_{0}$ be given by (3.6). Then for any $\psi \perp \varphi$,

$$
\left(\mathcal{F}_{\lambda_{0}} \psi\right)\left(z_{0}\right)=\overline{\left(1-\lambda_{0}\right) z_{0}} \overline{\left\langle\psi,\left(U-z_{0}\right)^{-1} \varphi\right\rangle}
$$

where $z_{0}=e^{i \theta_{0}}$ and (3.8) is shorthand for $\lim _{r \uparrow 1} \overline{\left\langle\psi,\left(U-r z_{0}\right)^{-1} \varphi\right\rangle}$, which it is asserted exists.

Proof. We use (2.14) for $z=r z_{0}$ and $\lambda=\lambda_{0}$. By the spectral theorem since $z_{0}$ is an eigenvalue of $U_{\lambda_{0}}$,

$$
\lim _{r \uparrow 1}(1-r)\left(U_{\lambda_{0}}-r z_{0}\right)^{-1} U_{\lambda_{0}} \varphi \equiv \eta_{z_{0}}
$$

is an eigenvector for $U_{\lambda_{0}}$ with eigenvalue $z_{0}$ or it is zero. Since (3.6) holds, we have that

$$
\begin{aligned}
\left\langle\varphi, \eta_{z_{0}}\right\rangle & =\lim _{r \uparrow 1} \frac{1-r}{2}\left[F_{\varphi}^{\lambda_{0}}\left(r z_{0}\right)+1\right] \\
& =\lambda_{0}\left[\frac{1-r}{\lambda_{0}-\left.[z f(z)]\right|_{z=r z_{0}}}\right] \quad \text { by (2.11) } \\
& =\frac{\lambda_{0} z_{0}}{\left(1-\lambda_{0} z_{0}\right)^{2}}\left(-G\left(z_{0}\right)\right)^{-1}
\end{aligned}
$$

by (10.1.7) of [16]. This is nonzero by the assumption $G\left(z_{0}\right)<\infty$. One can also use cyclicity of $\varphi$ to conclude that $\eta_{z_{0}} \neq 0$. Thus $\eta_{z_{0}} \neq 0$ and so, by (3.3) and (2.14),

$$
\left(\mathcal{F}_{\lambda} \psi\right)\left(z_{0}\right)=\lim _{r \uparrow 1} \frac{\overline{\left\langle\psi,\left(U-r z_{0}\right)^{-1} U \varphi\right\rangle}}{\overline{\left\langle\varphi,\left(U-r z_{0}\right)^{-1} U \varphi\right\rangle}}
$$


By (2.12) and $r z_{0} f\left(r z_{0}\right) \rightarrow \lambda_{0}$,

$$
\lim _{r \uparrow 1}\left\langle\varphi,\left(U-r z_{0}\right)^{-1} U \varphi\right\rangle=\left(1-\lambda_{0}\right)^{-1}
$$

and since $\left(\psi,(U-z)^{-1}(U-z) \varphi\right)=0$, we have

$$
\left\langle\psi,\left(U-r z_{0}\right)^{-1} U \varphi\right\rangle=r z_{0}\left\langle\psi,\left(U-r z_{0}\right)^{-1} \varphi\right\rangle
$$

Thus, we see that (3.9)-(3.11) implies (3.8).

Remark. In fact, (3.8) holds whenever $d \mu$ is purely singular and for all $\lambda_{0} \neq 1$. This is because $d \mu$ purely singular implies $d \mu_{\lambda}$ is purely singular and (3.9) can be replaced by Poltoratskii's theorem 13, 9, which says that for any complex Borel measure $\eta$ on $\partial \mathbb{D}$ and any $g \in L^{1}(\partial \mathbb{D}, d \eta)$, we have, for almost any $e^{i \theta_{0}}$ with respect to $d \eta_{\mathrm{s}}$ (but not for $d \eta_{\mathrm{ac}}$ ), that

$$
\lim _{r \uparrow 1} \frac{\left[\int \frac{e^{i \theta}+r e^{i \theta_{0}}}{e^{i \theta}-r e^{i \theta_{0}}} f(\theta) d \eta(\theta)\right]}{\left[\int \frac{e^{i \theta}+r e^{i \theta_{0}}}{e^{i \theta}-r e^{i \theta_{0}}} d \eta(\theta)\right]}=f\left(\theta_{0}\right)
$$

\section{Deterministic Form of Aizenman's Theorem}

We now follow Aizenman [1] and del Rio et al. [5]. Under the assumption that $\varphi$ is cyclic and $G\left(z_{0}\right)<\infty$ for a.e. $z_{0}$ in $\partial \mathbb{D}$, we have for $\psi \perp \varphi$ that for a.e. $\lambda_{0}$ that

$$
\left|\left(\mathcal{F}_{\lambda_{0}} \psi\right)\left(z_{0}\right)\right| \leq\left|\lim _{r \uparrow 1}\left\langle\varphi,\left(U+r z_{0}\right)\left(U-r z_{0}\right)^{-1} \psi\right\rangle\right|
$$

since $\psi \perp \varphi$ implies $\left\langle\varphi,(U+z)(U-z)^{-1} \psi\right\rangle=2 z\left\langle\varphi,(U-z)^{-1} \psi\right\rangle$. (4.1) holds for all eigenvalues of $U_{\lambda_{0}}$ and so, for a.e. $z_{0}$ w.r.t. $d \mu_{\lambda_{0}}$ if $U_{\lambda_{0}}$ is pure point.

Since $\mathcal{F}_{\lambda}$ is a unitary operator, we have

$$
\int\left|\left(\mathcal{F}_{\lambda_{0}} \psi\right)(z)\right|^{2} d \mu_{\lambda_{0}}(z)=\|\psi\|^{2}
$$

Moreover, since $\mathcal{F}_{\lambda_{0}} U \mathcal{F}_{\lambda_{0}}^{-1}=z$ and $\mathcal{F}_{\lambda_{0}} \varphi \equiv 1$,

$$
\begin{aligned}
\left|\left\langle\varphi, U_{\lambda_{0}}^{n} \psi\right\rangle\right| & =\left|\int z^{n} \mathcal{F}_{\lambda_{0}} \psi(z) d \mu_{\lambda_{0}}(z)\right| \\
& \leq \int\left|\mathcal{F}_{\lambda_{0}} \psi\left(z_{0}\right)\right| d \mu_{\lambda_{0}}(z)
\end{aligned}
$$

We conclude, using Hölder's inequality and (4.2):

Proposition 4.1. If $\varphi$ is cyclic and $G\left(z_{0}\right)<\infty$ for a.e. $z_{0}$, then for a.e. $\lambda_{0}$ and any $0<p<1$, we have

$$
\sup _{n}\left|\left\langle\varphi, U_{\lambda_{0}}^{n} \psi\right\rangle\right| \leq\left[\int \lim _{r \uparrow 1}\left|\left\langle\varphi,(U+r z)(U-r z)^{-1} \psi\right\rangle\right|^{p} d \mu_{\lambda_{0}}(z)\right]^{1 /(2-p)}
$$


Proof. We have

$$
\int|g| d \mu_{\lambda} \leq\left(\int|g|^{2} d \mu_{\lambda}\right)^{(1-p) /(2-p)}\left(\int|g|^{p} d \mu_{\lambda}\right)^{1 /(2-p)}
$$

since $(1-p) /(2-p)+1 /(2-p)=1$ and $2(1-p) /(2-p)+p /(2-p)=1$, and Hölder's inequality says $q \rightarrow \log \left(\int|g|^{q} d \mu\right)^{1 / q}$ is convex. (4.4) follows by taking $g=\mathcal{F}_{\lambda_{0}} \psi$ and using (4.1), (4.2), and (4.3).

Since $2-p>1$, for any probability measure $d \nu, \int h^{1 /(2-p)} d \nu \leq$ $\left(\int h d \nu\right)^{1 /(2-p)}$ by Hölder's inequality. Thus writing $\lambda_{0}=e^{i \eta_{0}}$ and integrating (4.4) with $d \eta_{0} / 2 \pi$, we find, using (3.7), that

Theorem 4.2 (Deterministic Aizenman's Theorem). If $\varphi$ is cyclic, $\psi \perp \varphi$, and $G\left(z_{0}\right)<\infty$ for a.e. $z_{0} \in \partial \mathbb{D}$, then for any $0<p<1$,

$$
\int \frac{d \eta}{2 \pi} \sup _{n}\left|\left\langle\varphi, U_{e^{i \eta}}^{n} \psi\right\rangle\right| \leq\left(\int \lim _{r \uparrow 1}\left|\left\langle\varphi,\left(U+r e^{i \theta}\right)\left(U-r e^{i \theta}\right)^{-1} \psi\right\rangle\right|^{p} \frac{d \theta}{2 \pi}\right)^{1 /(2-p)}
$$

\section{Rank One Perturbations of CMV Matrices}

We now specialize to $U=\mathcal{C}\left(\left\{\alpha_{j}\right\}_{j=0}^{\infty}\right)$, a CMV matrix (see [4] or [15, Chapter 4]), and $\varphi=\delta_{n}$, the vector with 1 in the $n$-th position ( $n=$ $0,1,2, \ldots)$. We need a notation for diagonal matrices with diagonal matrix elements $\lambda, \lambda^{-1}$, or 1 . $D\left(\lambda^{k}\left(\lambda^{-1}\right)^{\ell}(1 \lambda)^{\infty}\right)$ will denote the diagonal matrix with $k \lambda$ 's, $\ell\left(\lambda^{-1}\right)$ 's, and then alternating 1 and $\lambda$. We will also use the maps $T_{n, \lambda}$ of (1.5). Here is the main result:

Theorem 5.1. Define $U_{n}$ for $n=0,1,2, \ldots$ by

$$
\begin{aligned}
U_{2 k-1} & =D\left(1^{2 k}(1 \lambda)^{\infty}\right) \\
U_{2 k} & =D\left(\lambda^{2 k}(1 \lambda)^{\infty}\right)
\end{aligned}
$$

Then

$$
U_{n} \mathcal{C}\left(T_{n, \lambda^{-1}}(\alpha)\right) U_{n}^{-1}=\mathcal{C}(\alpha) \Delta_{n}(\lambda)
$$

where

$$
\Delta_{n}(\lambda)=D\left(1^{n} \lambda 1^{\infty}\right)
$$

Remarks. 1. Since $\mathcal{C}(\alpha) \Delta_{n}(\lambda)$ is unitary with $\delta_{0}$ cyclic, it is unitarily equivalent to some $\mathcal{C}(\tilde{\alpha})$. Since $\mathcal{C}(\alpha) \uparrow\left\{\delta_{j}\right\}_{j=0}^{n-1}=\mathcal{C}(\tilde{\alpha}) \uparrow\left\{\delta_{j}\right\}_{j=0}^{n-1}$, it is easy to see that $\tilde{\alpha}_{k}=\alpha_{k}$ for $k=0,1, \ldots, n-1$. By Khrushchev's formula ([16. Theorem 9.2.4]), the spectral measure for $\mathcal{C}(\alpha)$ and vector $\delta_{n}$ has Schur function $\Phi_{n}\left(z ; \alpha_{0}, \ldots, \alpha_{n-1}\right) \Phi_{n}^{*}\left(z ; \alpha_{0}, \ldots, \alpha_{n-1}\right)^{-1} f\left(z ; \alpha_{n}, \alpha_{n+1}, \ldots\right)$. By (2.11), $\tilde{\mathcal{C}}(\alpha)$ thus has Schur function which is this times $\lambda^{-1}$, so by Khrushchev's formula again, $f\left(z ; \tilde{\alpha}_{n}, \tilde{\alpha}_{n+1}, \ldots\right)=\lambda^{-1} f\left(z ; \alpha_{n}, \alpha_{n+1}, \ldots\right)=$ $f\left(z ; \lambda^{-1} \alpha_{n}, \lambda^{-1} \alpha_{n+1}, \ldots\right)$. We conclude $\tilde{\alpha}_{j}=\lambda^{-1} \alpha_{j}$ for $j \geq n$. (15.3) goes beyond this by making the unitary equivalence explicit.

2. The case $n=0$ is essentially Theorem 4.2 .4 of [15]. 
Proof. First, some preliminaries. We use $\oplus$ for direct sum, normally of $2 \times 2$ matrices but sometimes of a $1 \times 1$ followed by $2 \times 2$, in which case we write $\mathbf{1}_{1}$ or $\lambda \mathbf{1}_{1}$ so that, for example, if

$$
v(\lambda)=\left(\begin{array}{cc}
1 & 0 \\
0 & \lambda
\end{array}\right) \quad \tilde{v}(\lambda)=\left(\begin{array}{cc}
\lambda & 0 \\
0 & 1
\end{array}\right)
$$

then

$$
\begin{aligned}
U_{2 k-1} & =\underbrace{\mathbf{1}_{2} \oplus \mathbf{1}_{2} \oplus \cdots \oplus \mathbf{1}_{2}}_{k \text { times }} \oplus v(\lambda) \oplus v(\lambda) \oplus \cdots \\
& =\mathbf{1}_{1} \oplus \underbrace{\mathbf{1}_{2} \oplus \cdots \oplus \mathbf{1}_{2}}_{k \text { times }} \oplus \tilde{v}(\lambda) \oplus \tilde{v}(\lambda) \oplus \cdots
\end{aligned}
$$

If

$$
\Theta(\alpha)=\left(\begin{array}{rr}
\bar{\alpha} & \rho \\
\rho & -\alpha
\end{array}\right)
$$

with $\rho=\left(1-|\alpha|^{2}\right)^{1 / 2}$, then (see [15, Theorem 4.2.5])

$$
\begin{aligned}
\mathcal{C}(\alpha) & =\mathcal{L}(\alpha) \mathcal{M}(\alpha) \\
\mathcal{L}(\alpha) & =\Theta\left(\alpha_{0}\right) \oplus \Theta\left(\alpha_{2}\right) \oplus \Theta\left(\alpha_{4}\right) \oplus \cdots \\
\mathcal{M}(\alpha) & =\mathbf{1}_{1} \oplus \Theta\left(\alpha_{1}\right) \oplus \Theta\left(\alpha_{2}\right) \oplus \cdots
\end{aligned}
$$

Notice next that (note $v(\lambda)$ not $v(\lambda)^{-1}$ in both places!)

$$
\begin{aligned}
v(\lambda) \Theta\left(\lambda^{-1} \alpha\right) v(\lambda) & =\lambda \Theta(\alpha) \\
\tilde{v}(\lambda)^{-1} \Theta\left(\lambda^{-1} \alpha\right) \tilde{v}(\lambda)^{-1} & =\lambda^{-1} \Theta(\alpha)
\end{aligned}
$$

We now turn to the proof of (5.3) for $n=2 k-1$. By (5.6), (5.9), and (5.11),

$$
U_{2 k-1} \mathcal{L}\left(T_{2 k-1, \lambda^{-1}}(\alpha)\right) U_{2 k-1}=\lambda \mathcal{L}(\alpha) W
$$

where

$$
W=D\left(\left(\lambda^{-1}\right)^{2 k} 1^{\infty}\right)
$$

Now

$$
\begin{aligned}
W U_{2 k-1}^{-1} & =D\left(\left(\lambda^{-1}\right)^{2 k}\left(1 \lambda^{-1}\right)^{\infty}\right) \\
& =\lambda^{-1} \mathbf{1}_{1} \oplus \underbrace{\lambda^{-1} \mathbf{1}_{2} \oplus \cdots \oplus \lambda^{-1} \mathbf{1}_{2}}_{(k-1) \text { times }} \oplus \tilde{v}(\lambda)^{-1} \oplus \tilde{v}(\lambda)^{-1} \oplus \cdots
\end{aligned}
$$

since $\lambda^{-1}\left(1 \lambda^{-1}\right)^{\infty}=\left(\lambda^{-1} 1\right)^{\infty}$. Thus, by (5.12) and (5.10),

$$
W U_{2 k-1}^{-1} \mathcal{M}\left(T_{2 k-1, \lambda^{-1}}(\alpha)\right) U_{2 k-1}^{-1} W=\lambda^{-1} \mathcal{M}(\alpha) D\left(\left(\lambda^{-1}\right)^{2 k-1} 1^{\infty}\right)
$$

We conclude

$$
\begin{array}{rl}
U_{2 k-1} & \mathcal{C} \\
\quad & \left.T_{2 k-1, \lambda^{-1}}(\alpha)\right) U_{2 k-1}^{-1} \\
& =U_{2 k-1} \mathcal{L}\left(T_{2 k-1, \lambda^{-1}}(\alpha)\right) U_{2 k-1} U_{2 k-1}^{-1} \mathcal{M}\left(T_{2 k-1, \lambda^{-1}}(\alpha)\right) U_{2 k-1}^{-1} \\
& =\lambda \mathcal{L}(\alpha) W U_{2 k-1}^{-1} \mathcal{M}\left(T_{2 k-1, \lambda^{-1}}(\alpha)\right) U_{2 k-1}^{-1} W W^{-1} \quad(\text { by } \\
& =\mathcal{L}(\alpha) \mathcal{M}(\alpha) D\left(\left(\lambda^{-1}\right)^{2 k-1} 1^{\infty}\right) D\left((\lambda)^{2 k} 1^{\infty}\right) \quad(\text { by }
\end{array}
$$




$$
=\mathcal{C}(\alpha) \Delta_{n}(\lambda)
$$

since $\Delta_{n}(\lambda)=\left(1^{n} \lambda 1^{\infty}\right)$. This proves (5.3) for $n=2 k-1$.

Now suppose $n=2 k$. Then, by (5.11),

$$
U_{2 k} \mathcal{L}\left(T_{2 k, \lambda^{-1}}(\alpha)\right) U_{2 k}=\lambda \mathcal{L}(\alpha) \widetilde{W}
$$

where

$$
\widetilde{W}=D\left((\lambda)^{2 k} 1^{\infty}\right)
$$

Thus

$$
\begin{aligned}
\widetilde{W} U_{2 k}^{-1} & =D\left(1^{2 k}\left(1 \lambda^{-1}\right)^{\infty}\right) \\
& =\mathbf{1}_{1} \oplus \underbrace{\mathbf{1}_{2} \oplus \cdots \oplus \mathbf{1}_{2}}_{k \text { times }} \oplus \tilde{v}(\lambda)^{-1} \oplus \tilde{v}(\lambda)^{-1} \oplus \cdots
\end{aligned}
$$

This is the reason odd and even $n$ differ. (5.15) has $k-1 \mathbf{1}_{2}$ 's and (5.19) has $k$ of them. By (5.12) and (5.10),

$$
\widetilde{W} U_{2 k}^{-1} \mathcal{M}\left(T_{2 k, \lambda^{-1}}(\alpha)\right) U_{2 k}^{-1} \widetilde{W}=\lambda^{-1} \mathcal{M}(\alpha) D\left(\lambda^{2 k-1} 1^{\infty}\right)
$$

It follows that

$$
\begin{aligned}
U_{2 k} \mathcal{C}\left(T_{2 k, \lambda^{-1}}(\alpha)\right) U_{2 k} & =U_{2 k} \mathcal{L}\left(T_{2 k, \lambda^{-1}}(\alpha)\right) U_{2 k} U_{2 k}^{-1} \mathcal{M}\left(T_{2 k, \lambda^{-1}}(\alpha)\right) U_{2 k}^{-1} \\
& =\lambda \mathcal{L}(\alpha) \widetilde{W} U_{2 k}^{-1} \mathcal{M}\left(T_{2 k, \lambda^{-1}}(\alpha)\right) U_{2 k}^{-1} \widetilde{W} \widetilde{W}^{-1} \\
& =\mathcal{L}(\alpha) \mathcal{M}(\alpha) D\left(\lambda^{2 k+1} 1^{\infty}\right) D\left(\left(\lambda^{-1}\right)^{2 k} 1^{\infty}\right) \\
& =\mathcal{C}(\alpha) \Delta_{n}(\lambda)
\end{aligned}
$$

\section{Proof of Theorem 1.1}

We are now ready to put it all together:

Proof of Theorem 1.1. By (3.5) and the exponential decay in (1.6), $G\left(z_{0}\right)<$ $\infty$ for a.e. $\alpha$, so Theorem 4.2 applies for a.e. $\alpha$. By (4.5) with $\varphi=\delta_{k}$ and $\psi=\delta_{m}$ and $U=\mathcal{C}(\alpha)$

$$
\int \frac{d \varphi}{2 \pi} \sup _{n}\left|\left[\left(\mathcal{C}(\alpha) \Delta_{k}\left(e^{i \varphi}\right)\right)^{n}\right]_{k m}\right| \leq\left(\int\left|F_{k m}\left(e^{i \theta}\right)\right|^{p} \frac{d \theta}{2 \pi}\right)^{1 / 2}
$$

Now use Theorem 5.1 and the fact that $U$ is diagonal and unitary to see

$$
\left|\left[\left(\mathcal{C}(\alpha) \Delta_{k}(\lambda)\right)^{n}\right]_{k m}\right|=\left|\left[\mathcal{C}\left(T_{k, \lambda^{-1}}(\alpha)\right)^{n}\right]_{k m}\right|
$$

so that

$$
\int \frac{d \varphi}{2 \pi} \sup _{n}\left|\left[\mathcal{C}\left(T_{k, e^{-i \varphi}}(\alpha)\right)^{n}\right]_{k m}\right| \leq\left(\int\left|F_{k m}\left(e^{i \theta}\right)\right|^{p} \frac{d \theta}{2 \pi}\right)^{1 / 2}
$$

Take expectations of both sides. Use the quasi-invariance to write

$$
\mathbb{E}\left(\sup _{n}\left|\left[\mathcal{C}\left(T_{k, e^{-i \varphi}}(\alpha)\right)^{n}\right]_{k \ell}\right|\right) \geq C^{-1} \mathbb{E}\left(\sup _{n}\left|\left[C(\alpha)^{n}\right]_{k \ell}\right|\right)
$$


for a constant $C$ independent of $k, \ell, \varphi$. Use Hölder's inequality to bring $\mathbb{E}$ inside $(\cdot)^{1 /(2-p)}$. The result is

$$
\mathbb{E}\left(\sup _{n}\left|\left(\mathcal{C}^{n}\right)_{k \ell}\right|\right) \leq C\left(\int \mathbb{E}\left(\left|F_{k \ell}\left(e^{i \theta}\right)\right|^{p}\right) \frac{d \theta}{2 \pi}\right)^{1 / 2}
$$

which shows (1.6) implies (1.7).

\section{REMARKS}

Some closing remarks:

1. It is not hard to prove a local version of this theorem where $\int_{0}^{2 \pi}$ in (1.6) is replaced by $\int_{a}^{b}$ and an extra $P_{(a, b)}(\mathcal{C})$ is added in (1.7). This might be useful in the quasi-invariant case, but in the i.i.d. rotation invariant case, $\mathbb{E}\left(\left|F_{k \ell}\left(e^{i \theta}\right)\right|^{p}\right)$ is $\theta$-invariant and so the integral has exponential decay for $(a, b)$ if and only if it does for $(0,2 \pi)$.

2. If $d \rho$ is a rotation quasi-invariant measure on $\mathbb{D}$ and $d \gamma$ one on $\partial \mathbb{D}$, and if $\alpha_{0}, \bar{\alpha}_{0} \alpha_{1}, \bar{\alpha}_{1} \alpha_{2}, \ldots$ are independent random variables with $\alpha_{0} d \rho$-distributed and each $\bar{\alpha}_{j} \alpha_{j+1}, d \rho$-distributed, then this measure is quasi-invariant. It would be interesting to do localization theory (both spectral and dynamic) for this model.

3. It would be interesting to know if (1.7) implies (1.6).

\section{REFERENCES}

[1] M. Aizenman, Localization at weak disorder: Some elementary bounds, Rev. Math. Phys. 6 (1994), 1163-1182.

[2] M. Aizenman and S. Molchanov, Localization at large disorder and at extreme energies: An elementary derivation, Comm. Math. Phys. 157 (1993), 245-278.

[3] N. Aronszajn and W.F. Donoghue, On exponential representations of analytic functions in the upper half-plane with positive imaginary part, J. Analyse Math. 5 (1957), $321-388$.

[4] M.J. Cantero, L. Moral, and L. Velázquez, Five-diagonal matrices and zeros of orthogonal polynomials on the unit circle, Linear Algebra Appl. 362 (2003), 29-56.

[5] R. del Rio, S. Jitomirskaya, Y. Last, and B. Simon, Operators with singular continuous spectrum, IV. Hausdorff dimensions, rank one perturbations, and localization, J. Analyse Math. 69 (1996), 153-200.

[6] P.L. Duren, Theory of $H^{p}$ Spaces, Pure and Applied Math., 38, Academic Press, New York-London, 1970.

[7] Ya. L. Geronimus, Orthogonal Polynomials: Estimates, Asymptotic Formulas, and Series of Polynomials Orthogonal on the Unit Circle and on an Interval, Consultants Bureau, New York, 1961.

[8] L. Golinskii and P. Nevai, Szegő difference equations, transfer matrices and orthogonal polynomials on the unit circle, Comm. Math. Phys. 223 (2001), 223-259.

[9] V. Jakšić and Y. Last, A new proof of Poltoratskii's theorem, J. Funct. Anal. 215 (2004), 103-110.

[10] S. Khrushchev, Schur's algorithm, orthogonal polynomials, and convergence of Wall's continued fractions in $L^{2}(\mathbb{T})$, J. Approx. Theory 108 (2001), 161-248. 
[11] A. Kolmogorov, Sur les fonctions harmoniques conjuguées et les séries de Fourier, Fund. Math. 7 (1925), 24-29.

[12] N. Minami, Local fluctuation of the spectrum of a multidimensional Anderson tight binding model, Comm. Math. Phys. 177 (1996), 709-725.

[13] A.G. Poltoratskii, The boundary behavior of pseudocontinuable functions, St. Petersburg Math. J. 5 (1994), 389-406.

[14] B. Simon, Spectral analysis of rank one perturbations and applications, in "Proc. Mathematical Quantum Theory, II: Schrödinger Operators" (edited by J. Feldman, R. Froese, and L. Rosen), CRM Proc. Lecture Notes 8 (1995), 109-149.

[15] B. Simon, Orthogonal Polynomials on the Unit Circle, Vol. 1: Classical Theory, AMS Colloquium Series, American Mathematical Society, Providence, RI, in press.

[16] B. Simon, Orthogonal Polynomials on the Unit Circle, Vol. 2: Spectral Theory, AMS Colloquium Series, American Mathematical Society, Providence, RI, in press.

[17] B. Simon and T. Wolff, Singular continuous spectrum under rank one perturbations and localization for random Hamiltonians, Comm. Pure Appl. Math. 39 (1986), 75-90.

[18] M. Stoiciu, in preparation.

[19] G. Szegő, Orthogonal Polynomials, Amer. Math. Soc. Colloq. Publ., Vol. 23, American Mathematical Society, Providence, R.I., 1939; 3rd edition, 1967. 